\title{
Editorial
}

\section{Heated humidification: a simple question but ...}

as passive insulation using blankets to cover exposed skin or the use of heated water mattress or a convective air warmer to decrease the temperature gradient for heat exchange. Even at $50 \%$ efficiency these measures would have been equally effective and probably less costly than a heated humidifier in preventing the body heat changes noted in the control subjects.

During the first hour after operation on average the estimated total body heat of the control subjects increased by $229 \mathrm{~kJ}$ equivalent to an oxygen consumption rate of $189 \mathrm{ml} \cdot \mathrm{min}^{-1}$ averaged over one hour. Whether a postoperative heat deficit will provoke a metabolic response sufficiently severe to harm patients will depend on the amount of rewarming which has occurred when the residual narcosis dissipates. Thus any degree of heat deficit at the end of anaesthesia might under certain circumstances prove deleterious. The finding that external auditory temperature had not regained preinduction levels after one hour recovery suggests that in this particular group of subjects metabolic heat production was not accelerated.

In the humidified group one may estimate that with a similar amount of room temperature fluids administered and a similar area of exposed skin losing heat there was an even greater heat loss from these sources because of the higher skin and core temperature (Table). Basal metabolism will have contributed more because of the longer duration of surgery. In order to account for the overall average heat loss of $44 \mathrm{~kJ}$ a total respiratory gain of $408 \mathrm{~kJ}$ would be required. However, the maximum direct heat gain due to humidified (RH 140\%) gas delivered to the patient at $40^{\circ} \mathrm{C}$ would be $40 \mathrm{~kJ}$. As Gilmour et al. ${ }^{2}$ have noted, however, the Bennett Cascade humidifier also produces an aerosol of water droplets. To gain the remaining $368 \mathrm{~kJ}$, about $22 \mathrm{~kg}$ of water would have to cool from 40 to $36^{\circ} \mathrm{C}$ inside the body. Even under the most favourable circumstances the mass of water required to account for the difference between the control and humidified patients would be at least $9 \mathrm{~kg}$. However, this discrepancy between apparent and direct heat gains is not unique, ${ }^{3}$ and as was pointed out previously, ${ }^{4}$ if 
TABLE Average conditions and estimated partial heat balances based on average values of the patients studied by Kulkarni et al. ${ }^{1}$

\begin{tabular}{llllllllll}
\hline & $\begin{array}{l}\text { Ambient } \\
{ }^{\circ} \mathrm{C}\end{array}$ & $\begin{array}{l}\text { Skin } \\
{ }^{\circ} \mathrm{C}\end{array}$ & $\begin{array}{l}\text { Duration } \\
\mathrm{hr}\end{array}$ & $\begin{array}{l}\text { Core } \\
{ }^{\circ} \mathrm{C}\end{array}$ & $\begin{array}{l}\text { Respiration } \\
k J\end{array}$ & $\begin{array}{l}\text { Metabolism } \\
k J\end{array}$ & $\begin{array}{l}\text { Skin heat } \\
k J\end{array}$ & $\begin{array}{l}\text { Infusion } \\
k J\end{array}$ & $\begin{array}{l}\text { Balance } \\
k J\end{array}$ \\
\hline Control & 20.1 & 32.5 & 1.9 & 34.8 & -44 & 460 & -644 & -123 & -350 \\
Humidity & 19.8 & 33.8 & 2.25 & 36 & 408 & 545 & -861 & -135 & -44 \\
\hline
\end{tabular}

one accepts the validity of the temperature measurements then the only remaining hypothesis is that heated and humidified gases increase metabolic heat production.

What if anything may one conclude? Heated humidification apparently alters the heat balance of surgical patients in some fashion which is as yet undetermined. Fully defining the contribution of any active heating device used to prevent or treat surgical hypothermia requires a careful assessment and possibly measurement of all the sources heat gain or loss to which patients are exposed. In addition passive heat retention measures which can be applied to control groups such as the insulation of exposed skin surfaces not directly involved in surgery and respiratory heat and moisture exchangers need to be considered. Finally in today's cost-conscious environment no such technology assessment can be considered complete without the economic costs and the risks $^{2}$ of active vs control heat conservation measures being taken into account. Should we use heated humidification to prevent heat loss during peripheral surgery is as simple a question as it is possible to ask, unhappily there are no simple answers.

\section{L'humidification chauffée? Une question bien simple, mais ...}

Pendant l'anesthésie, le réchauffement de l'humidité compense-t-il efficacement pour l'accroissement de la perte calorique? Ce mois-ci Kulkarni, Webster et Carli ${ }^{1}$ répondent un oui à cette question apparemment toute simple. Chez un groupe de dix patients, opérés pour une prothèse totale de la hanche, ils ont trouvé que, comparativement au groupe contrôle, les gaz humidifiés par la chaleur diminuent considérablement la température du conduit auditif externe et la température cutanée moyenne pendant la chirurgie. Avec les variations de température constatées pendant la chirurgie ainsi qu'une heure après, ils évaluent, pendant la chirurgie, les variations caloriques moyennes de l'organisme à une perte nette de $350 \mathrm{~kJ}$ chez le groupe contrôle et de $44 \mathrm{~kJ}$ chez le groupe réchauffement de l'humidité. De plus, pendant la première heure postopératoire, le groupe contrôle retient $229 \mathrm{~kJ}$ de chaleur présumée endogène alors que le groupe humidité réchauffée n'en retient que $57 \mathrm{~kJ}$. Pour porter un jugement de valeur sur ces résultats en clinique, on devrait se demander normalement quelles méthodes de rechange auraient pu être utilisées pour prévenir la perte de chaleur chez les sujets du groupe contrôle et si cette perte est réellement dommageable.

Chez les sujets du groupe contrôle, la chaleur est perdue par la respiration, par l'exposition de surfaces cutanée et par la perfusion de liquides à la température ambiante. Le gain calorique d'origine métabolique pendant $1,9 \mathrm{~h}$ est de $460 \mathrm{~kJ}$ pour une consommation en oxygène $\left(\mathrm{VO}_{2}\right)$ horaire de $200 \mathrm{ml} \cdot \min ^{-1}$. Pendant la même période, les auteurs évaluent à $44 \mathrm{~kJ}$ la perte par la ventilation normocapnique avec des gaz secs (HR 10\%) administrés par un circuit à rebreathing et à $123 \mathrm{~kJ}$ la perte due à la perfusion de deux litres de liquide à la tempérture ambiante (Tableau).

Parce que la chaleur corporelle totale baisse de 350 $\mathrm{kJ}$, il s'ensuit que le sujet moyen aura perdu la balance de $644 \mathrm{~kJ}$ par rayonnement et par convection par la peau non couverte. Comme la peau non couverte perd de la chaleur à la vitesse d'environ $36 \mathrm{~kJ} \cdot \mathrm{h}^{-1} \cdot \mathrm{m}^{-2} \cdot{ }^{\circ} \mathrm{C}^{-1}$, on peut estimer que la surface disponible pour l'échange calorique équivaut à $0,76 \mathrm{~m}^{2}$ de peau exposée. Plusieurs anesthésistes ont l'habitude de prendre des mesures destinées à diminuer la perte de chaleur par la peau, telles que l'isolation passive avec des couvertures sur la peau exposée ou par l'usage d'un matelas chauffant à l'eau ou à convection d'air dans le but de diminuer le gradient de température disponible pour l'échange de la chaleur. Même avec un niveau d'efficacité de 50\%, ces mesures auraient été aussi efficaces et, selon toutes probabilités, moins coûteuses qu'un humidificateur-réchauffeur pour prévenir les variations de chaleur constatées dans le groupe contrôle.

Au cours de la première heure qui suit lintervention, en moyenne, la perte de chaleur corporelle totale du groupe contrôle augmente de $229 \mathrm{~kJ}$, ce qui équivaut à une consommation en oxygène de $189 \mathrm{ml} \cdot \mathrm{min}^{-1}$. Que le déficit calorique puisse provoquer une réponse métabolique nocive grave dépendra du degré de réchauffement 
TABLEAU Conditions moyennes et balances caloriques estimées basées sur les valeurs moyennes des patients inclus dans l'étude de Kulkarni $e t$ al. ${ }^{\prime}$

\begin{tabular}{|c|c|c|c|c|c|c|c|c|c|}
\hline & $\begin{array}{l}T^{\circ} a m b \\
C^{\circ}\end{array}$ & $\begin{array}{l}T^{\circ} \mathrm{cut} . \\
\mathrm{C}^{\circ}\end{array}$ & $\begin{array}{l}\text { Durée } \\
H\end{array}$ & $\begin{array}{l}T^{\circ} \text { cent. } \\
C^{\circ}\end{array}$ & $\begin{array}{l}\text { Resp. } \\
\text { kJ }\end{array}$ & $\begin{array}{l}\text { Métab. } \\
k J\end{array}$ & $\begin{array}{l}\text { Chal. cut. } \\
k J\end{array}$ & $\begin{array}{l}\text { Perfusion } \\
k J\end{array}$ & $\begin{array}{l}\text { Balance } \\
\mathrm{kJ}\end{array}$ \\
\hline Contrôle & 20,1 & 32,5 & 1,9 & 34,8 & -44 & 460 & -644 & -123 & -350 \\
\hline Humidité & 19,8 & 33,8 & 2,25 & 36 & 408 & 545 & -861 & -135 & -44 \\
\hline
\end{tabular}

atteint lorsque la narcose résiduelle sera dissipée. Ainsi, le déficit calorique présent à la fin de l'anesthésie pourrait, dans certaines circonstances, s'avérer nuisible. Le fait que la température du conduit auditif externe ne soit pas revenue à son niveau pré-induction une heure après le réveil suggère que, dans ce groupe particulier de patients, la production de chaleur par métabolisme ne s'est pas accélérée.

Dans le groupe humidité réchauffée, on peut estimer qu'avec une quantité identique de liquide administrée à la temperature ambiante et avec la même surface d'exposition cutanée, la perte calorique par ces sources est encore plus importante parce que les températures cutanées et centrales sont plus élevées. La contribution du métabolisme de base aura été plus importante à cause de la durée de la chirurgie. Un gain total de $408 \mathrm{~kJ}$ par la respiration est nécessaire pour compenser pour une perte moyenne totale de $44 \mathrm{~kJ}$. Cependant le gain direct maximum possible avec les gaz humidifiés (HR 140\%) fournis à $40^{\circ} \mathrm{C}$ serait $40 \mathrm{~kJ}$. Comme Gilmour et al. ${ }^{2}$ l'ont déjà fait remarquer, l'humidificateur Bennett Cascade émet des gouttelettes en aérosol. Pour regagner le déficit de $368 \mathrm{~kJ}$, environ $22 \mathrm{~kg}$ d'eau devront refroidir de 40 à $36^{\circ} \mathrm{C}$ à l'intérieur de l'organisme. Même dans les circonstances les plus favorables, la masse d'eau requise pour faire disparaître la différence entre les patients du groupe contrôle et ceux du groupe humidité serait d'au moins $9 \mathrm{~kg}$. Cependant, cette disparité apparente avec les gains de chaleur n'est pas unique ${ }^{3}$ comme il a déjà été noté; ${ }^{4}$ si on accepte la validité des mesures de température, la seule hypothèse qui subsiste, est que les gaz chauffés et humidifiés augmentent la production de la chaleur métabolique.

Quelle conclusion peut-on tirer de cette étude? Lhumidification réchauffée semble altérer la balance calorique du patient d'une façon encore indéterminée. La détermination exacte de la contribution d'un dispositif actif de réchauffement utilisé dans le but de prévenir ou de traiter l'hypothermie pendant la chirurgie nécessite une évaluation soignée et complète des sources de gain et perte caloriques auxquelles les patients sont exposés. On outre, les méthodes passives de compensation applicables à des groupes contrôles telles que l'isolation de la surface cutanée exposée intouchée par la chirurgie, et l'efficacité des échangeurs de chaleur et d'humidité doivent être prises en considération. Finalement à une époque où les coûts prennent une importance capitale, aucune évaluation de technologie ne peut être complète sans l'évaluation des coûts et des risques ${ }^{2}$ qui s'appliquent aux moyens de conservation calorique actifs et passifs. Devrions-nous utiliser l'humidification chauffée pour prévenir un déficit calorique pendant une chirurgie périphérique peut sembler une question bien simple, mais malheureusement la réponse ne l'est pas.

\section{References}

1 Kulkarni P, Webster J, Carli F. Body heat transfer during hip surgery using active core warming. Can J Anaesth 1995; 42: 571-6.

2 Gilmour IJ, Boyle MJ, Rozenberg A, Palahniuk RJ. The effect of heated wire circuits on humidification of inspired gases. Anesth Analg 1994; 79: 160-4.

3 Stone DR, Downs JB, Paul WL, Perkins HM. Adult body temperature and heated humidification of anesthetic gases during general anaesthesia. Anesth Analg 1981; 60: 736-41.

4 Hendrickx $H H L$, Trahey GE. Paradoxical inhibition of decreases in body temperature by the use of heated and humidified gases. Anesth Analg 1982; 61: 393-4. 